\title{
Templated Mesoporous Silica Colloids with Controlled Internal Structures
}

\section{Weon-Sik Chae and Paul V. Braun}

University of Illinois at Urbana-Champaign, Urbana, Illinois 61801, USA

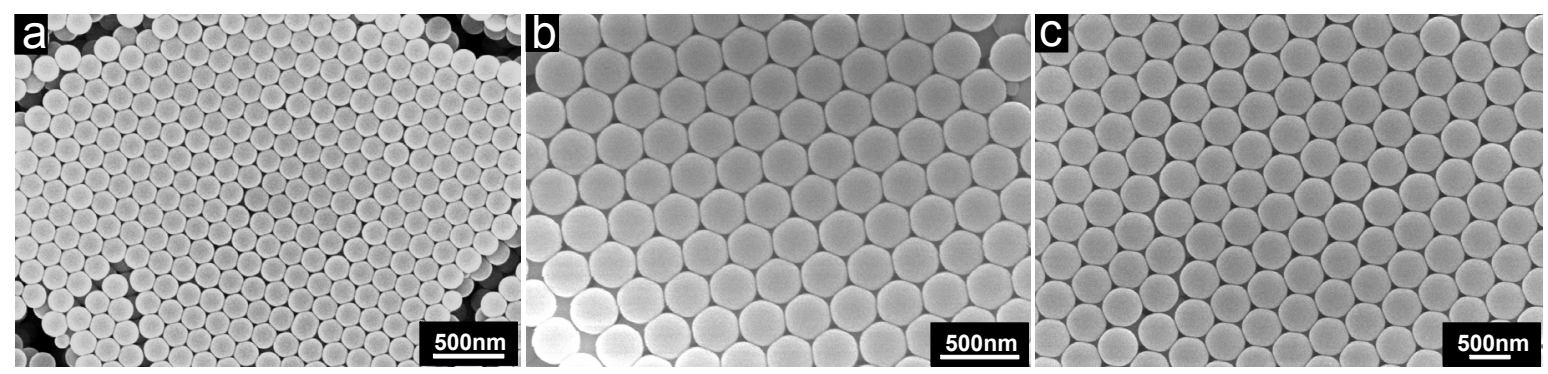

Figure S1. SEM images of the colloidal silica particles of different diameters: (a) 178, (b) 298, and (c) 498 $\mathrm{nm}$.
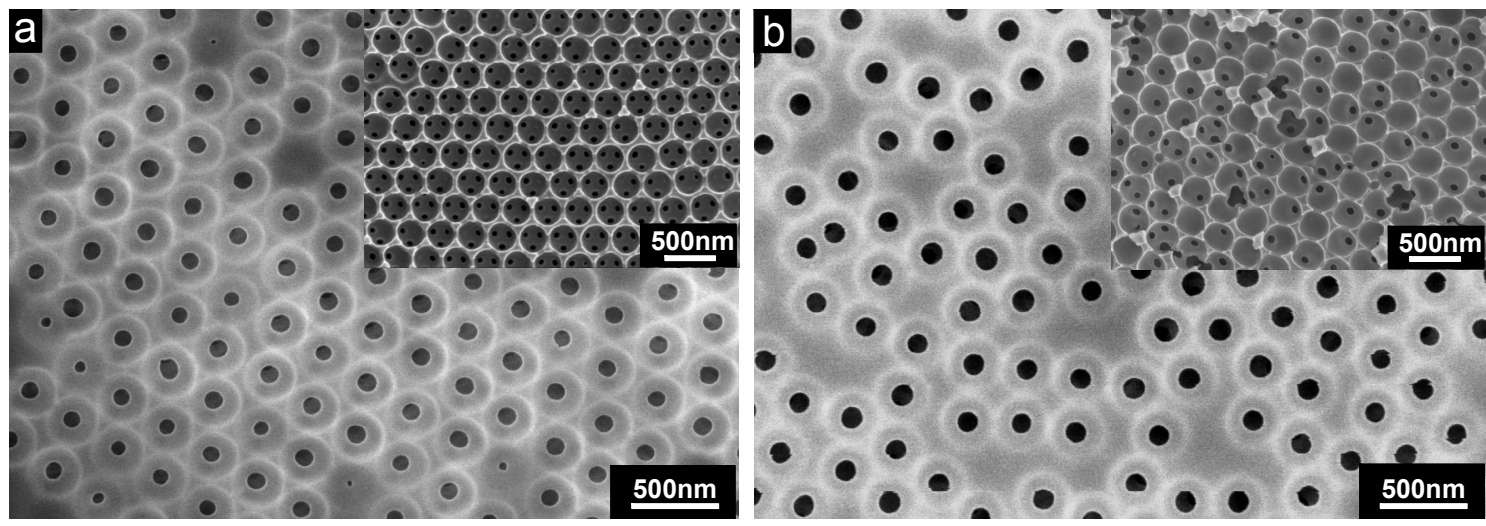

Figure S2. SEM images of (a) ordered and (b) disordered inverse opals with a cavity diameter of $260 \mathrm{~nm}$. The images are taken at the interface between the polymer inverse opal and glass slide (previously removed). Insets are cross sectional views of the corresponding inverse opals.
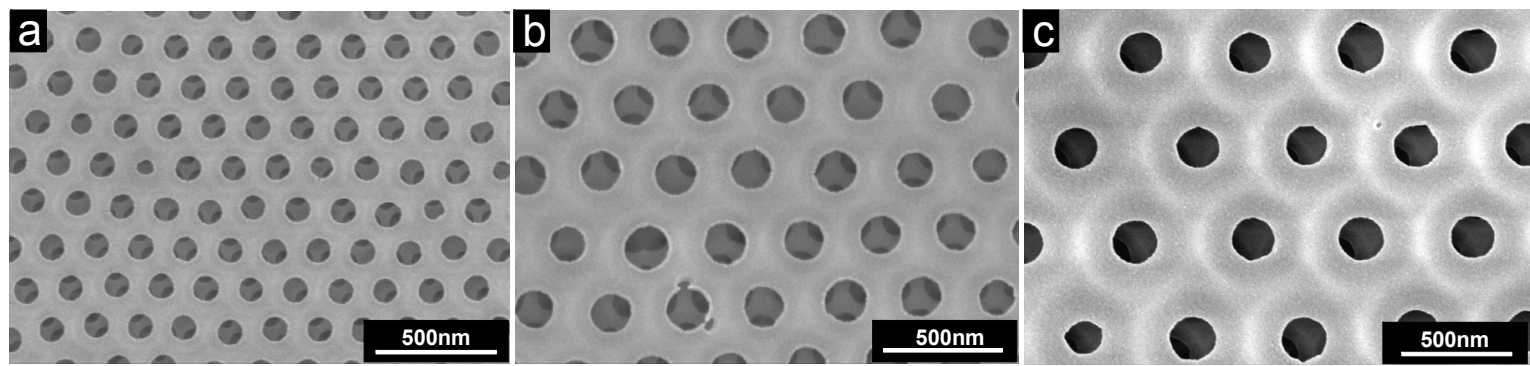

Figure S3. SEM images of the oxygen plasma treated polymer inverse opals of different cavity diameters: (a) 150, (b) 260, and (c) $451 \mathrm{~nm}$. The images are taken at the interface between the polymer inverse opal and the glass slide (previously removed). 